2018-06-01

\title{
Advancing Raman Microspectroscopy for Cellular and Subcellular Analysis: Towards in Vitro High Content Spectralomic Analysis
}

Hugh Byrne

Technological University Dublin, hugh.byrne@tudublin.ie

Franck Bonnier

Université Francois-Rabelais de Tours

Alan Casey

Technological University Dublin, alan.casey@tudublin.ie

See next page for additional authors

Follow this and additional works at: https://arrow.tudublin.ie/biomart

Part of the Biological and Chemical Physics Commons

\section{Recommended Citation}

Byrne, H.J., Bonnier, F. \& Alan Casey. (2018). Advancing Raman microspectroscopy for cellular and subcellular analysis: Towards in vitro high content spectralomic analysis. Applied Optics, 57, (22) E11-E19 (2018) doi:10.1364/A0.57.000E11

This Article is brought to you for free and open access by the Biomedical and Environmental Sensing at ARROW@TU Dublin. It has been accepted for inclusion in Articles by an authorized administrator of ARROW@TU

Dublin. For more information, please contact

arrow.admin@tudublin.ie, aisling.coyne@tudublin.ie, gerard.connolly@tudublin.ie.

Funder: SFI

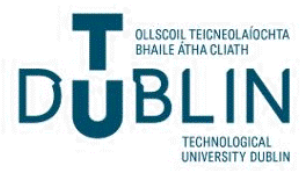




\section{Authors}

Hugh Byrne, Franck Bonnier, Alan Casey, Marcus Maher, Jennifer Mclntyre, Esen Efeoglu, and Zeineb Farhane 


\title{
Advancing Raman microspectroscopy for cellular and subcellular analysis: Towards in vitro high content spectralomic analysis
}

\author{
Hugh J. Byrne, ${ }^{1,}{ }^{*}$ Franck Bonnier, ${ }^{2}$ Alan Casey, ${ }^{3}$ Marcus Maher, ${ }^{1}$ Jennifer \\ MCINTYRe, ${ }^{1}$ ESEn EfEOglu, ${ }^{1,3}$ ZeIneb FARHANE ${ }^{1,3}$ \\ ${ }^{1}$ FOCAS Research Institute, Dublin Institute of Technology, Kevin Street, Dublin 8, Ireland \\ ${ }^{2}$ Université François-Rabelais de Tours, Faculty of Pharmacy, EA 6295 Nanomédicaments et Nanosondes, 31 avenue Monge, 37200 Tours, \\ France. \\ ${ }^{3}$ School of Physics and Optometric \& Clinical Sciences, Dublin Institute of Technology, Kevin Street, Dublin 8, Ireland \\ *Corresponding author: hugh.byrne@dit.ie
}

Received XX Month XXXX; revised XX Month, XXXX; accepted XX Month XXXX; posted XX Month XXXX (Doc. ID XXXXX); published XX Month XXXX

In the confocal mode, Raman microspectroscopy can profile the biochemical content of biological cells at a subcellular level, and any changes to it by exogenous agents, such as therapeutic drugs or toxicants. As an exploration of the potential of the technique as a high content, label free analysis technique, this report reviews work to monitor the spectroscopic signatures associated with the uptake and response pathways of commercial chemotherapeutic agents and polymeric nanoparticles by human lung cells. It is demonstrated that the signatures are reproducible and characteristic of the cellular event, and can be used, for example, to identify the mode of action of the agent as well as the subsequent cell death pathway, and even mechanisms of cellular resistance. Data mining approaches are discussed and a spectralomics approach is proposed.

OCIS codes: (170.5660) Raman spectroscopy, (180.5655) Raman microscopy, (170.1530) Cell analysis, (170.1580) Chemometrics.

http://dx.doi.org/10.1364/AO.99.099999

\section{INTRODUCTION}

The application of Raman spectroscopy to biomolecules and even tissues was first demonstrated as early as the 1960s[1-3] and by the mid-1970s biomedical applications were explored[4]. Whole cell and tissue studies have been carried out on a range of pathologies[5-9] and in vivo studies[10-12] have demonstrated the prospective for diagnostic applications. The potential of vibrational spectroscopy in conjunction with multivariate analysis techniques as a diagnostic tool has thus been well demonstrated and the concept of Spectral Cytopathology has been coined[13]. However, since the original claims in the 1990s, clinical translation has been slow, potentially due to demands of large scale clinically relevant studies and the rigours of legislative approval, prompting an appraisal of more realistic strategically achievable targets for the field[14]. Importantly, the intrinsic strength of spectroscopy is in materials and process analysis rather than classification, and in vitro studies of cells allow a detailed analysis of the fundamental cell biology, or biochemical changes, for example as a result of an external agonist, toxicant or chemotherapeutic agent[15-17]. The technique is label free, and, in the case of Raman microspectroscopy, subcellular analysis can be performed in the confocal microscopic mode, providing high content spectroscopic signatures of biochemical profiles, promising potentially more realisable applications in fundamental cellular (cytological) research, medicinal chemistry and pharmacological/toxicological screening. Regulatory requirements in both the EU and US (EU Directive-2010/63/EU and US Public Law 106-545, 2010, 106th Congress) have increasingly restricted the use of animal models for development of pharmaceuticals and cosmetics, and Raman microspectroscopy offers a potentially low cost, label free alternative to in vitro High Content Analysis for routine screening.[18, 19]

This paper reviews recent work carried out at the Dublin Institute of Technology, to advance Raman microspectroscopy for cellular and subcellular analysis, probing the limitations of the technique in terms of reproducibility and specificity of spectral signatures of subcellular events and pathways. Specifically, it explores the areas of (A) Cellular differentiation (B) Drug screening and (C) Nanotoxicological screening. As models, the responses elicited by established and commercially available chemotherapeutic agents and nanoparticle standards in commercially available in vitro cell lines are chosen. The often 
subtle spectroscopic changes associated with the cellular perturbations are elucidated using a range of multivariate data mining techniques, and the challenge of identifying consistent reproducible label free spectral markers is addressed.

\section{EXPERIMENTAL}

The details of experimental methods and protocols are described in the original publications[20-23], but are summarised here.

\section{A. Materials}

A549 human lung adenocarcinoma cells with the alveolar type II phenotype were obtained from ATTC (Manassas, VA, USA) and Calu-1 human lung epidermoid carcinoma cells were obtained from the European Collection of Cell Cultures.

Doxorubicin hydrochloride $\AA$ powder (Sigma Life Sciences, Ireland) was diluted in $1 \mathrm{~mL}$ sterile water to the required concentration, identified by cytotoxicological assays.

$40 \mathrm{~nm}$ non-toxic carboxyl-modified polystyrene nanoparticles (PSNPs) were employed to determine nanoparticle localisation and trafficking, whereas $100 \mathrm{~nm}$ toxic amine-modified polystyrene nanoparticles (PS- $\mathrm{NH}_{2}$ ) were used to determine spectral markers of the toxicity. Generation 5 PAMAM dendrimers were used as a secondary toxic nanoparticle model for comparison of spectral markers of toxicity. All nanomaterials were purchased from Sigma-Aldrich (Ireland).

\section{B. Cell Culture}

A549 cells were cultured in DMEM (with $2 \mathrm{mM} \mathrm{L-glutamine)} \mathrm{with}$ $10 \%$ foetal bovine serum (FBS) and Calu- 1 cells in RPMI with $10 \%$ FBS, both at $37^{\circ} \mathrm{C}$ in a humidified atmosphere containing $5 \% \mathrm{CO}_{2}$ and cells were split every two days to maintain $\sim 60 \%$ confluence.

\section{Raman Microspectroscopy}

Cells $\left(\sim 3 \times 10^{3} / \mathrm{cm}^{2}\right)$ were seeded and incubated on $(20 \mathrm{~mm}$ diameter) $\mathrm{CaF}_{2}$ windows (Crystan Ltd, UK) for $24 \mathrm{hrs}$ for both control and exposure to chemotherapeutic drug or nanoparticles. Medium was then removed and samples were rinsed twice with sterile PBS and covered with solutions of the appropriate drug solution or nanoparticle suspension concentration. After each incubation period cells were washed twice with sterile PBS and fixed in formalin (10\%,15 min).

A Horiba Jobin-Yvon LabRAM HR800 spectrometer with a $785 \mathrm{~nm}$, $300 \mathrm{~mW}$ diode laser as source, Peltier cooled 16-bit CCD, 300 lines $/ \mathrm{mm}$ grating and $100 \mu \mathrm{m}$ confocal hole, was used for this work. Cells were measured either air dried or in water immersion, and spectra were acquired from the three cell locations: cytoplasm, nuclear and nucleolar in the range from $400 \mathrm{~cm}^{-1}$ to $1800 \mathrm{~cm}^{-1}$ with an x100 objective (LCPlanN, Olympus (dry), or LUMPlanF1, Olympus (immersion)) for 30 s two times, to finally produce a data set of 30 points per cell location for each cell line, over a total of 90 different cells.

\section{Data Processing and Analysis}

Raman spectral pre-processing and analysis were performed in Matlab using algorithms developed in house. Prior to analysis, background was subtracted using a NCLS (non-negatively constrained least squares) algorithm, spectra were smoothed (Savitsky-Golay filter 3th order, 11 points), baseline corrected (fifth order polynomial) and vector normalised.
Principal components analysis (PCA) was employed as an unsupervised multivariate approach to analyse data and the effects of the drug or nanoparticles in each cell localisation. The order of the PCs denotes their importance to the dataset and PC1 describes the highest amount of variation.

Partial least squares regression (PLSR), a statistical regression technique which reduces the dimensionality of the data and correlates information, here represented by the Raman spectra, to values of the drug concentration inside the cells, and the time evolution, was employed to track the temporal and dose dependent evolution of the spectral signatures in the subcellular regions. The percentage of variance explained as a function of the number of components was calculated using 10-fold cross validation and from a plot of the percent of variance explained as a function of number of components, the majority percentage of variance (above $90 \%$ ) was found to be explained by the first 3 components.

The regression coefficients obtained by PLSR of Raman data regressed separately against drug cellular concentration and time evolutions were plotted and analysed. As a function of frequency, the coefficients illustrate the spectral features which are influenced by the intracellular interaction of the drug and the resulting metabolic changes.

\section{Results}

\section{A. Cellular differentiation}

Raman spectra were taken from the three cellular regions for all cell lines and Figure 1 shows the average spectra corresponding to each cell region, for the example of A549 cells [20]. Visibly, it can be seen that the mean spectra of the nucleolar, nuclear and cytoplasmic regions are somewhat different, and discriminating peaks (indicated by highlighted regions in Figure 1) at, for example, 1578, 1095, 830 and $795 \mathrm{~cm}^{-1} \mathrm{can}$ be assigned to nucleic acids, corresponding respectively to vibrations of the DNA bases adenine and guanine, DNA $\mathrm{PO}_{2}$ - symmetric stretching, ribose phosphate and DNA backbone O-P-O stretching, [24, 25] while others at 1300 ( $\mathrm{CH}$ deformation) and $717 \mathrm{~cm}^{-1}\left(\mathrm{CN}+\left(\mathrm{CH}_{3}\right)_{3}\right.$ stretching) are associated with lipid components of the cell membrane, which are also observable in the spectra of the nuclear and nucleolar regions, although more prominently in the spectra of the cytoplasmic region. The DNA bands at $1095 \mathrm{~cm}^{-1}$ and $830 \mathrm{~cm}^{-1}$ indicate that the DNA is in the $\mathrm{B}$ form, while the peak at $813 \mathrm{~cm}^{-1}$ corresponds to DNA A form.[26]

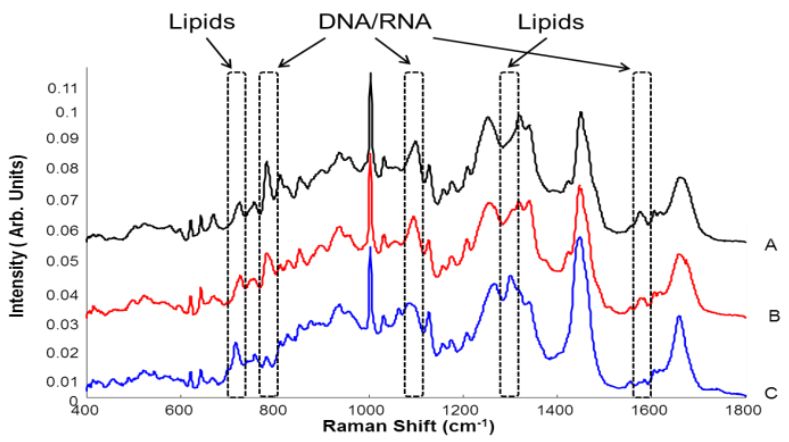

Fig. 1. Mean spectra of A. Nucleolus, B. Nucleus and C. Cytoplasm of A549 cell line. Highlighted regions correspond to DNA/RNA and lipids features.[20]

For a more detailed understanding of the differences in spectral profiles of the subcellular regions, a multivariate analysis is more informative, and therefore, PCA was employed to analyse and compare the spectral profiles of the subcellular 
regions as well as the differences between cancer and normal cell lines. Figure 2 shows the PCA scatter plot of the cytoplasmic, nuclear and nucleolar spectra of the Calu-1 cell line, along with the corresponding loadings of PC1 and PC2. PC1 clearly differentiates between the cytoplasmic and integrated nuclear regions (nuclear and nucleolar) and, according to the corresponding loading (loading 1 ), the most visible discriminant features derive from DNA, $795 \mathrm{~cm}^{-1}$ and $1095 \mathrm{~cm}^{-1}$, related to BDNA O-P-O backbone stretching and $\mathrm{PO}_{2}-$ symmetric stretching, and lipids at $717\left(\mathrm{CN}+\left(\mathrm{CH}_{3}\right)_{3}\right.$ stretching $), 1300\left(\mathrm{CH}_{2}\right.$ stretching) and $1440 \mathrm{~cm}^{-1}$ ( $\mathrm{CH}$ stretching). The differentiation of the subcellular regions according to PC1 is not unexpected, due to the significant biochemical differences between the combined nuclear and cytoplasmic regions which are similar for all cell lines. The nuclear and nulceolar regions can be differentiated by a further, pairwaise PCA of the two subcellular regions.[20]

Notably, a similar differentiation between the subcellular regions of A549 cells can be performed, and the differentiating PC loadings are almost identical to those of Calu-1 (Figure 2C). In fact, although they are relatively well distinguished from normal cell lines, differentiation between cancer cell lines is only achievable by comparison of the nucleolar regions.[22] Critically, the similarities of the differentiating subcellular spectral profiles for different cell lines supports the consistency and robustness of the technique for subcellular analysis.
A.

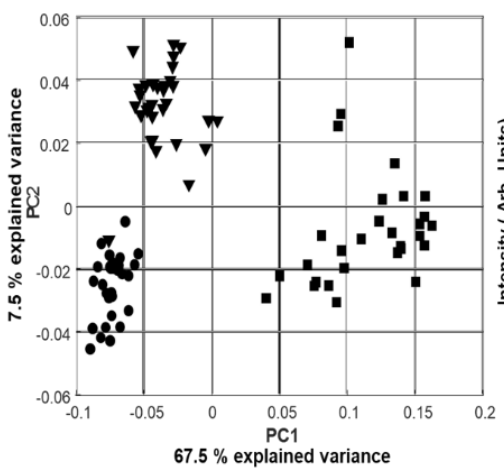

B.

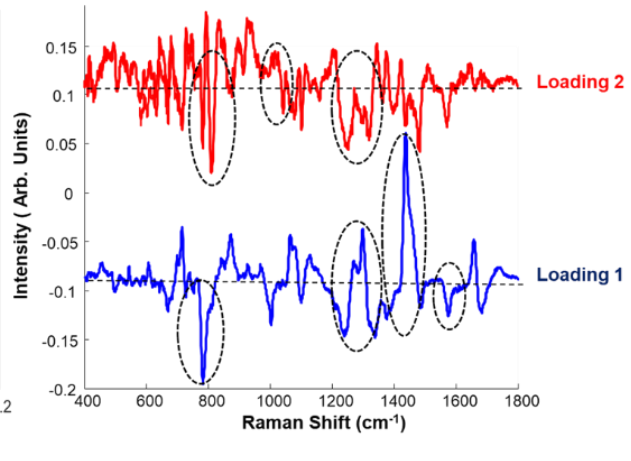

C.

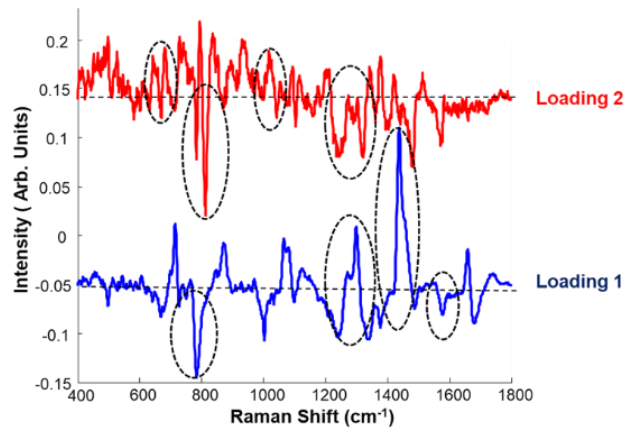

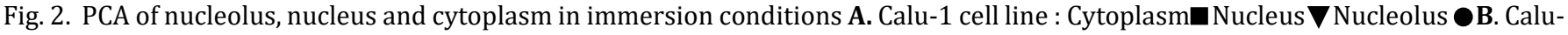
1 differentiating PC loadings C. A549 differentiating PC loadings [20]

\section{B. Drug screening}

Raman microspectroscopy has attracted considerable attention over the last few years in the pharmaceutical field to aid in areas such as the drug discovery process, quality control during industrial manufacturing and detection of counterfeit products,[27-29] as well as its possible clinical applications.[30, 31 Its potential as a screening technique either for normal and cancer cell discrimination or in chemotherapeutic and cellular process screening has been demonstrated.[32]

Doxorubicin (DOX), a non cell cycle specific chemotherapeutic agent, is one of the two first anthracycline antibiotics isolated from Streptomyces peucetius[33] and among the most widely used chemotherapeutic agents, despite its numerous side effects, $[34,35]$ and is highly effective in treating different types of cancers, from leukaemia, thyroid, and lung to many other neoplasia, especially metastatic and invasive ones.[36-38] The accepted mechanisms of action of DOX are complex and not fully understood. They include interactions within the cell nucleus, consisting of DNA intercalation, crosslinking, binding and alkylation, inhibition of topoisomerase I and II and RNA polymerase, all inducing DNA damage, inhibition of DNA replication and protein synthesis (rRNA transcription inhibition), as a consequence, leading to nucleolar disruption and cellular apoptosis[39]. DOX also interacts with the cell membrane and mitochondria, resulting in generation of reactive oxygen species leading to direct membrane damage and oxidative stress, responsible for the major DOX side effect, cardiomyopathy.[35, 40-42] DNA remains the main target of cancer therapeutics, whereby induction of DNA damage initiates a cascade of events that determines cellular apoptosis. DNA damage level and repair, by expression of anti-apoptotic proteins such as bcl-2 and resistance to apoptosis, are the main processes involved in carcinogenesis and in the response of cancer cells to cancer chemotherapy.[43]

A.

$$
\text { B. }
$$
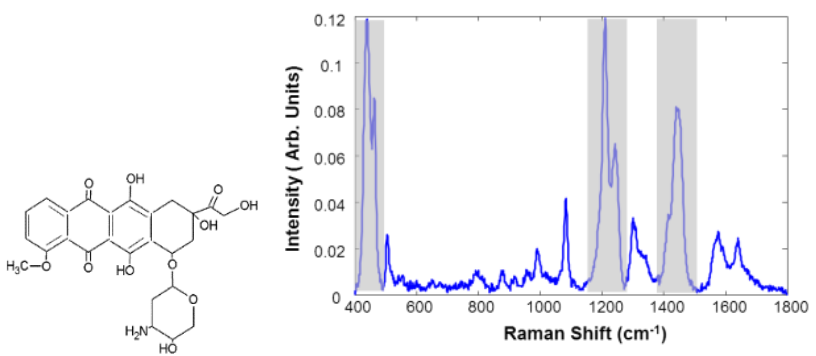

Fig. 3. A. Structure of Doxorubicin B. Raman spectrum of in aqueous solution indicating strong DOX marker bands.[21]

To demonstrate the potential of Raman microspectroscopy to monitor the uptake of chemotherapeutic agents, to track the drug pathway and monitor cellular responses, Raman profiles from the three subcellular compartments, nucleolus, nucleus and cytoplasm, were analysed as a function of time after exposure of cell cultures to DOX sulutions. The anthracycline structure of DOX (Figure 3A), consisting of a conjugated anthraquinone ring structure banded to an aminoglycoside [44], 
renders it fluorescent and gives it a strong Raman scattering efficiency even off resonantly, (Figure 3B).

The characteristic spectral profile of DOX can be easily identified superimposed on that of the exposed cell lines, and Figure 4A shows the sucellular uptake of DOX in the A549 and Calu- 1 cell lines, as represented by the characteristic band at 465 $\mathrm{cm}^{-1}$ [21] There is a progressive increase of DOX intracellular concentration until a plateau is reached after 6-12 hrs exposure and DOX is seen to accumulate in the nucleolus first, then in the nucleus and after 24-48 hrs appears in the cytoplasm. The behaviour is consistent with saturation of nuclear binding sites after a certain time and any additional drug taken up by cells accumulates in the cytoplasm.[45, 46] Interestingly, the accumulation rates are substantially different for the two cell lines investigated. Simultaneously, the characteristic cellular features of the subcellular regions can be tracked, as shown in Figure 4B, for the example of the nucleolus.

A.
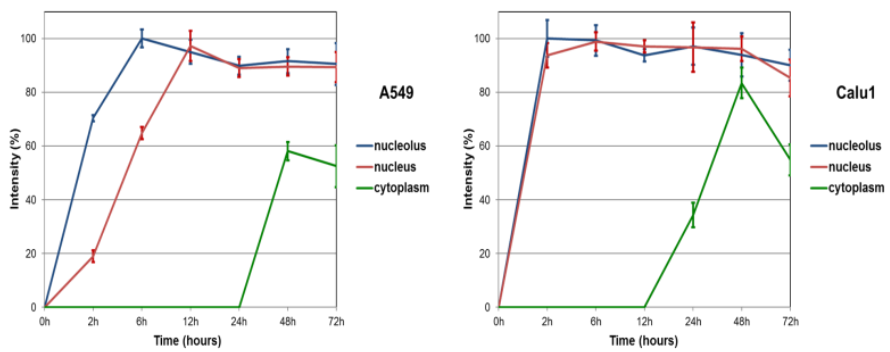

B.
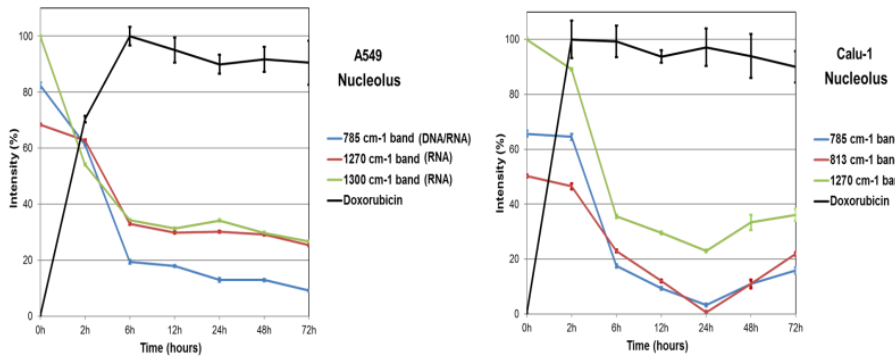

Fig. 4. A. Evolution of DOX, represented by the Raman band at $465 \mathrm{~cm}^{-1}$, as a function of time for the A549 and Calu-1 cell line for each cellular compartment, nucleolus, nucleus and cytoplasm. B. Evolution of selected DNA and RNA features as function of time. Intensities are expressed as percentage according to the maximum value over the three cellular compartments for each cell line and standard deviation corresponds to the spectral variations of the Raman band over the 30 measurements per location. $[49,50]$

Multivariate PLSR can be employed to profile the spectrum of the initial binding interactions of the drug in the subcellular regions, and the subsequent cellular responses of the cells, in the plateau regions. There is a striking similarity between the responses of the different cell-lines to the same drug, and indeed of the cell lines to drugs of similar mode of action, as demonstrated for Actinomycin D (Act), a commonly employed anthracyclic chemotherapeutic agent which is similar in molecular structure and mode of action to DOX.(47) Comparing the spectroscopic signatures with the commonly clinically employed chemotherapeutic agents Cisplatin (Cisp) and Vincristine (Vinc), there is a remarkable consistency of spectroscopic signatures of nucleic acids in the nuclear regions, as shown in Figure 5.(48)

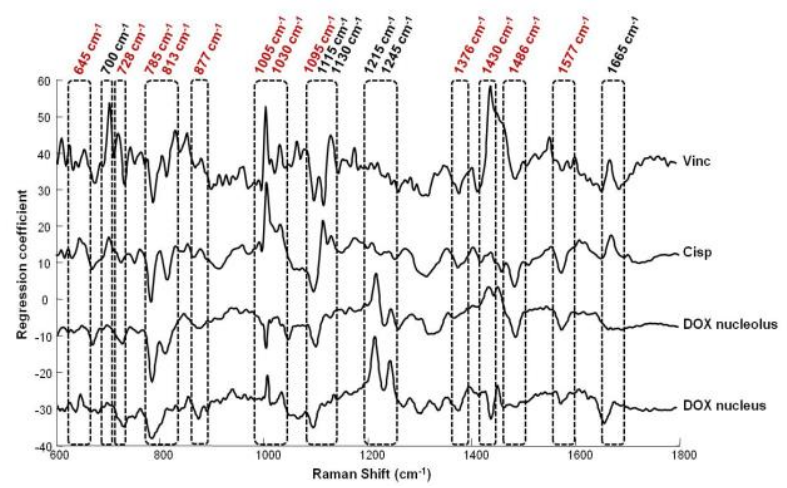

Fig. 5. Regression coefficients obtained by PLSR analysis for the A549 cell line as a function of drug dose for chemotherapeutic drugs DOX, Cisp (nucleus) and Vinc (nucleus). [50]

The study of the intracellular localization of DOX demonstrates that the primary subcellular target is in fact the nucleolus, in which it interacts strongly with the RNA. Using confocal microscopy, upon DOX exposure, the nucleoli of the cell appear dark, however, indicating that the characteristic fluorescence of DOX is quenched in this dense subcellular region. Therefore, Raman microspectroscopy sheds light on the cellular interactions and medicinal chemistry, in situ, which were not previously known. In the case of the structurally similar ACT, the cytotoxicologically relevant dose is so low that neither the fluorescence not the characteristic Raman signature can be traced at a cellular level. Nevertheless, the initial binding interactions of the drug within the nucleolus and nucleus of the cell can be identified, associating them with the similar mode of action of intercalation as DOX. The timescales of the responses are significantly slower, however, in both cell lines, which can be understood in terms of the different intracellular trafficking processes associated with the bulky polypeptide sidechains of ACT, compared to DOX. Raman microspectroscopy also demonstrates that, although Cisp and Vinc are reported to have different modes of action, as alkylating agents and microtubule binders, respectively, their Raman signatures evolve from low to high doses. At doses above their median inhibitory concentrations, the signatures also exhibit characteristics of intercalation, as shown DOC and ACT, indicating that they can have multiple modes of action.[48]

Monitoring the time evolution of drug distribution within the cell, the spectroscopic signatures of the chemical interaction in the subcellular regions can be identified, and associated with the characteristic modes of action of the drug. The signatures are consistent for drugs of similar modes of action, and thus can be exploited as a rapid in vitro tool for pre-screening of candidate drugs and a guide to design strategies for chemotherapeutics. The subsequent evolution of the spectroscopic signatures can then be associated with the cellular response pathways. Clear differences can be seen in the response pathways of the A549 and Calu-1 cell-lines,[47] as evidenced in the univariate analysis of Figure 4B. PLSR analysis, and correlation with assays to probe the generation of anti-apoptotic proteins and DNA repair, as mechanisms of cellular defense, indicate that the differences in the Raman signatures at the longer time points may be associated with differing degrees of cellular resistance.[47] 


\section{Nanotoxicological screening}

Most of the strategies that have been proposed for the investigation of nanomaterials include the combinational use of toxicity assays, bio-imaging techniques, chemical and biochemical assays, to obtain information regarding to metabolic activity, membrane integrities, cellular viability and cell death mechanisms upon exposure. Cytotoxicity assays have been widely employed to determine cellular viability and also cell death rate in vitro. Electron microscopy (EM) and optical microscopy have been used for the visualisation of nanomaterials in cells. Confocal Microscopy (CLSM) has also been used for the visualisation of nanomaterials in cells with the aid of fluorescent labels.[51-54] High Content Analysis/Screening (HCA/HCS), has opened up new perspectives for toxicological assessment by providing multifaceted information with high sensitivity based on automated imaging of 'phenotypic assay endpoints'. The technique has been used for in vitro toxicity assessment, animal disease models, drug discovery, neurobiology,[55, 56] cell signalling[57-59] and observation of biochemical changes.[58, $60,61]$ However, although conventional techniques have provided invaluable information regarding the interaction of nanomaterials with living systems, they remain limited in various aspects, such as the requirement of multiple labels and assays, interference of nanomaterials with the assay dyes,[6264] cost and also time required for the investigation. Raman microspectroscopy offers the potential to holistically screen uptake mechanisms of, and cellular responses to, nanoparticles, in vitro.

The correlation between Raman spectral markers of carbon nanotubes (CNTs) and commonly used cytotoxicity assays has been studied by Knief et al. [17]. The studies of Dorney et al. [65] and Keating et al. [66] have shown the localisation of nanoparticles in cells and the use of multivariate analysis techniques to obtain more detailed information regarding to subcellular environment. As an extension of these studies, Efeoglu et al. demonstrated that the spectroscopic signatures of the nanoparticle environment in the cytoplasm can be tracked, as they are processed through endosomes ( $4 \mathrm{hrs}$ ), to lysosomes (12hrs), to the endoplasmic reticulum (24hrs), for the case of 40 nm non-toxic carboxyl-modified PSNPs, as shown in Figure 6.[22] Expressed in terms of the loadings of the principal component which differentiates the nanoparticle local environment from control, the signatures demonstrate clear differences between the biochemical compositions, largely in terms of lipidic and protein content.

For the case of $100 \mathrm{~nm}$ toxic PS- $\mathrm{NH}_{2}$, the biochemical composition of the cytoplasm as a whole is perturbed by the cellular responses to exposure, and can be monitored by Raman microspectroscopy. PS- $\mathrm{NH}_{2}$ are known to elicit acute toxicity as a result of oxidative stress in the endosomal and lysosomal pathway [22, 23, 65]. Figure 7 shows the loadings of PCA obtained from pairwise comparison of particle exposed cells (24 hrs) and corresponding controls. Positive and negative features of the loadings relate to exposed and unexposed cells, respectively and the figure summarises spectral markers of acute toxic response in the cytoplasm, identified by Raman microspectroscopy. The predominant spectral marker of the toxic response in the cytoplasm is observed to be the 'doublet band' at 785 and $810 \mathrm{~cm}^{-1}$, which indicates a change in the cytoplasmic RNA as a result of ROS formation and the doublet band is observed in particle exposed cells even at low doses

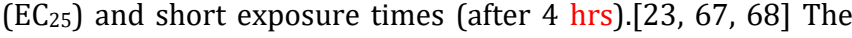
intensity of the band systematically and progressively changed as a function of dose and exposure time [23]. The doublet band was also observed in the biochemical features of Calu- 1 cells following $\mathrm{PS}-\mathrm{NH}_{2}$ exposure and progressively changed with extended exposure times [67]. When a second toxic nanoparticle model, PAMAM dendrimers, was introduced to the A549 cells, a similar trend to that for PS- $\mathrm{NH}_{2}$ is observed for the double peak at 785 and $810 \mathrm{~cm}^{-1}$, which, together with the results obtained from different cell lines and nanoparticle models, validates the changes in cytoplasmic RNA as a spectral marker of toxicity [68]..

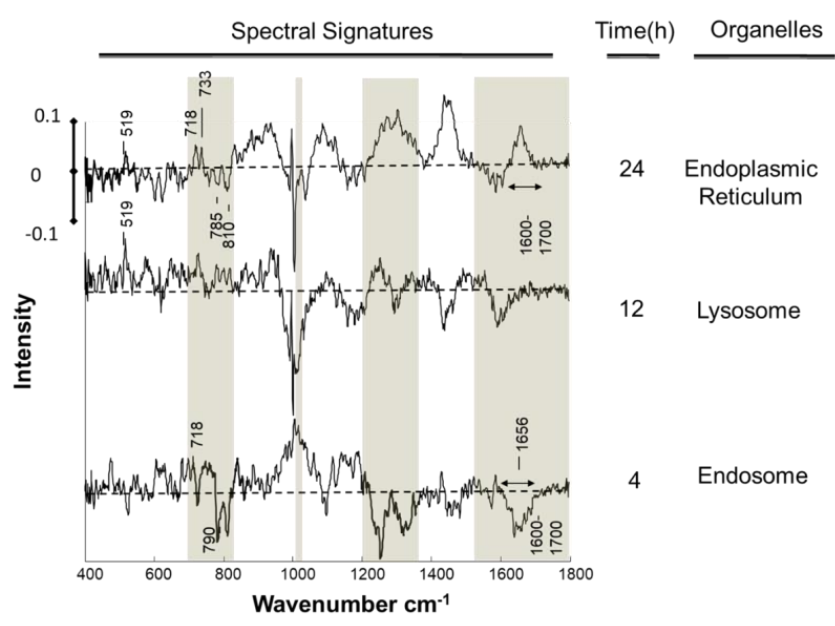

Fig. 6. Comparison of PCA loadings of 4, 12 and 24 hrs nanoparticle exposure data sets. Loadings are offset for clarity. The dotted line represents the zero '0' point for each loading and an intensity scale of $0 \pm 0.1$ is used for comparison. [22].

The changes in the doublet band, upon exposure to $\mathrm{PS}-\mathrm{NH}_{2}$ and PAMAM, are followed by concomitant and subsequent changes in the higher end of the fingerprint region, which provides information about protein (Amide I region) and lipid damage (1229 and $1438 \mathrm{~cm}^{-1}$ ). The band at $1438 \mathrm{~cm}^{-1}$, which has been identified upon PS- $\mathrm{NH}_{2}$ exposure, was also identified after exposure to PAMAM dendrimers and it has been observed to provide information regarding to lipotoxicity [23, 67, 69]. Notably, however, especially at extended exposure times, a significantly different evolution of spectral markers has been observed for the two different types of toxic nanoparticles, which can be attributed to different cell death mechanisms. Although the changes were more significant at long exposure times, the different response rates of the bands at early time points also provided information regarding the different cell death mechanisms. Cell death upon toxicant exposure can occur in multiple ways, most notably apoptosis and necrosis. The conventional apoptosis/necrosis assays use colorimetric identification based on cellular uptake of dyes and give information related to whether apoptotic or necrotic cell death has occurred. However, Raman microscopy not only provides information regarding whether the cell is apoptotic or necrotic, but also the biochemical path leading to this differentiation (Figure 8).

Therefore, it can be concluded that Raman microspectroscopy identifies signatures of the initiating toxic response and subsequent cellular dependent events, as a single, real-time assay and notably, brings new perspectives to 
determination of nanomaterial toxicity by identifying cytoplasmic RNA as a spectral marker, which is not normally identified by conventional toxicity assays. Moreover, the doublet band can be seen as the first strong spectroscopic response of oxidative stress. Although, it does not measure the ROS directly, it provides information about the response, which is a ROS related change in cytoplasmic RNA. The onset of ROS formation upon nanoparticle exposure has been observed to be within 2-6 hrs $[69,70]$, and this initial stage of ROS dies away due to the action of intracellular antioxidants with a similar timescale [69]. In contrast, the doublet band is observed only after $8 \mathrm{hrs}$ exposure to $\mathrm{PS}-\mathrm{NH}_{2}$ and increases further with exposure time.

Adopting the terminology of the Adverse Outcome Pathway methodology [71], upon exposure to a toxicant, the response begins with a molecular initiating event (MIE) which induces a cascade of consequent events. In the current context, the MIE is identified as the initial oxidative stress caused by the ingestion of the nanoparticles in exosomes and ultimately results in a change in cytoplasmic RNA, manifest as changes in the doublet peak of $785 \mathrm{~cm}^{-1}$ and $810 \mathrm{~cm}^{-1}$. The study of Maher et al.[72] showed that the MIE shows particle dependency and induces cellular dependant events which can include, for example, changes in mitochondrial function, activation of caspases, and release of inflammatory factors. However, conventional and commonly used cytotoxicity assays use specific markers which can be defined as 'endpoints' and identify the toxicity as a function of half-maximal effective dose ' $\mathrm{EC}_{50}$ '. Therefore, these assays remain limited to provide information regarding to multiple events that occur concomitantly or consecutively inside the cell. Raman microspectroscopy shows signatures of this cascade as presence of spectral markers of initiating toxic event at low doses and short exposure times in the low wavenumber region (cytoplasmic RNA, indirect ROS), followed by concomitant changes (Protein and lipid damage as a result of cellular toxic events) in the higher wavenumber region of the fingerprint.

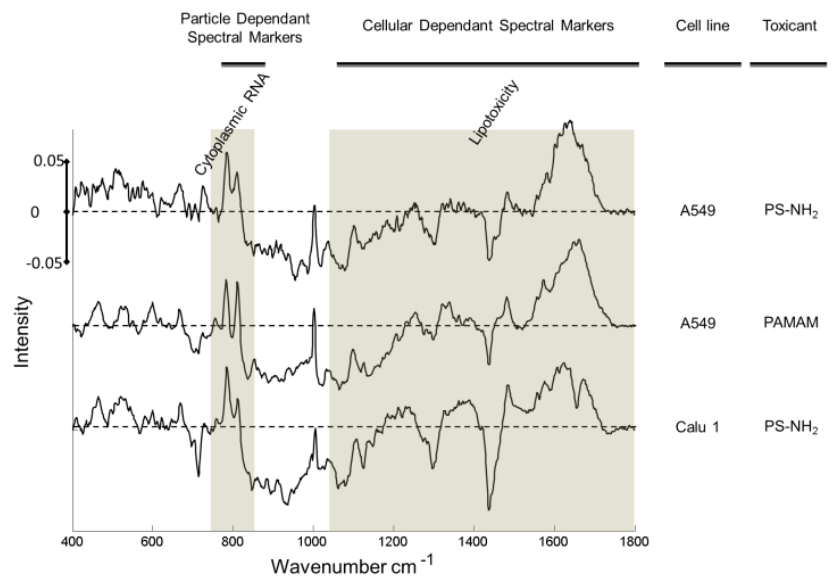

Fig. 7. Spectral Markers of acute toxic response in the cytoplasm of A549 and Calu- 1 cells after 24 hrs exposure to PS- $\mathrm{NH}_{2}$ and PAMAM nanoparticles. Positive and negative features of the loadings relate to exposed and unexposed cells, respectively. The $750-830 \mathrm{~cm}^{-1}$ and above $1000 \mathrm{~cm}^{-1}$ region are indicated with highlights. Loadings are offset for clarity. The dotted line represents the zero ' 0 ' point for each loading and intensity scale of $0 \pm 0.05$ is used for comparison $[23,67,68]$.
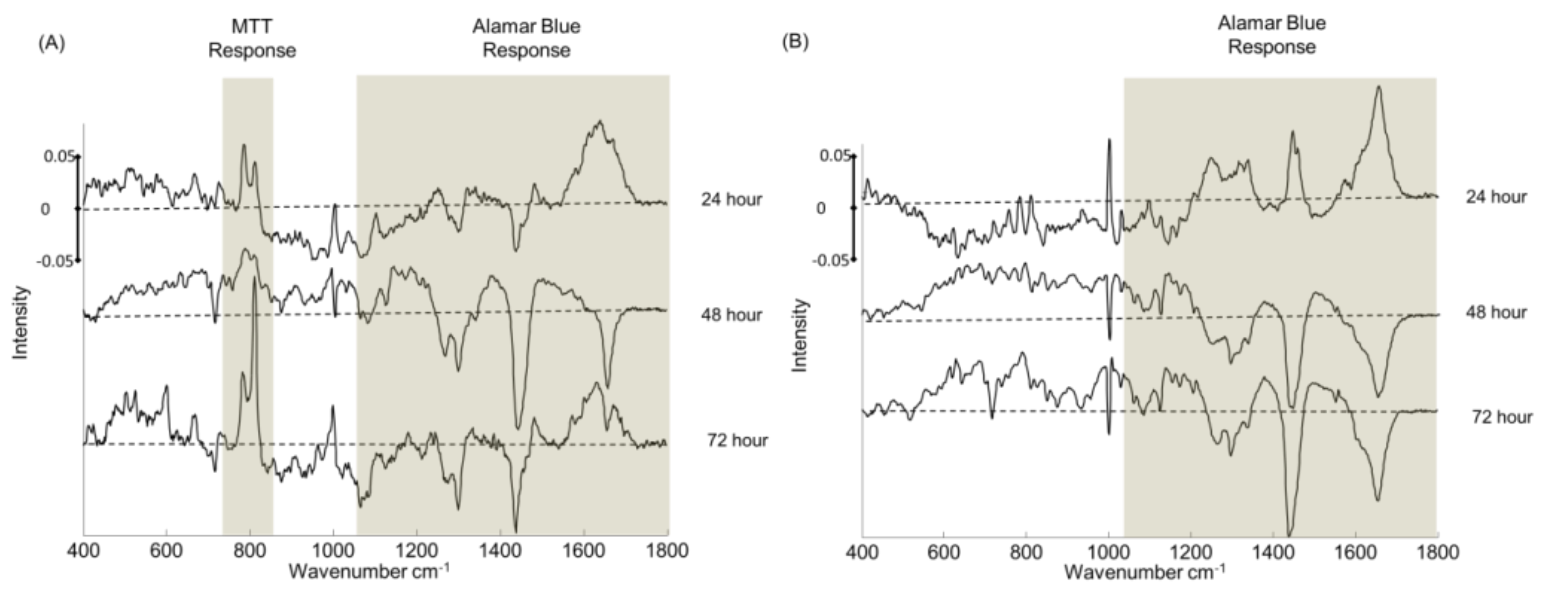

Fig. 8. Spectral markers of cell death on cancerous (A) and non-cancerous (B) cell lines and cytotoxicity assays which are found to be related to the changes of these spectral markers. Loadings are offset for clarity. The dotted line represents the zero ' 0 ' point for each loading and intensity scale of $0 \pm 0.05$ is used for comparison. [65].

\section{Discussion and Conclusions}

Technological advances have rendered Raman confocal microspectroscopy an increasingly routine benchtop laboratory technique for materials and process analysis. As an optical technique, it can provide submicron spatial resolution and can image biological cells and functions at a subcellular level, providing a wealth of information about the biochemical makeup, and changes to it due to exogeneous agents. The studies described herein were designed to explore the reproducibility of the characteristic spectroscopic signatures associated with cellular uptake of such agents and the subsequent response pathways.

The results clearly demonstrate that the subcellular regions of the cell can be reproducibly differentiated based on their intrinsic biochemistry, and that the characteristic spectroscopic profiles are consistent across different cell lines. The subcellular localisation of chemotherapeutic agents or nanoparticles can be determined, and characteristic signatures of their initial 
chemical interactions can be established. The signatures are characteristic of the mode of action of the chemotherapeutic agent or nanoparticle, and, even in cases where the signatures of the agents themselves cannot be identified (at low doses or for weak Raman scatterers), these characteristic signatures can act as evidence of the action of the agent. These initial spectroscopic signatures can be differentiated from those of the subsequent cellular response pathways, which can specifically indicate the mode of cell death or cellular defence mechanisms.

The studies of the model chemotherapeutic agent DOX demonstrate a clear added value of the technique, as they extend the current understanding of the mode of action by revealing the importance of the accumulation in the cell nucleoli, interacting with the RNA and initiating a cascade of cellular responses. In fluorescence based confocal microscopy, the nucleoli of DOX exposed cells appear dark, compared to the strongly fluorescent, DOX laden surrounding nucleus, and so it is understandable that such nucleolar based mechanisms have been overlooked, to date. Raman microspectroscopy reveals, however, that the nucleolar regions are equally laden with the drug, although it appears that the fluorescence is quenched due to the binding interactions in the dense region of the cell.

The label free nature of the technique can thus be of great benefit for fundamental biochemical and cellular biological research. It enables, for example, the in situ monitoring of candidate drugs, of benefit to medicinal chemistry, and the ability to monitor the processes in live cells can provide a wealth of high content information for modelling approaches to pharmacokinetics and (nano) toxicology at a cellular level. The recent emergence of nonlinear Raman microspectroscopic techniques adds significantly to the potential in the field, as video rate, full spectrum analyses of areas of hundreds of microns have already been reported.[73] Applications in toxicological screening to guide regulatory processes, in vitro pre-clinical candidate drug screening to guide synthetic strategies, and even patient screening for drug sensitivity/resistance as the basis for companion diagnostics, can readily be envisaged.

It should be noted, however, that a critical remaining challenge is that of data-mining the spectral information which is gathered. Label free, "what you see is what you get", and the characteristic spectral signature of a response pathway contains information of all biomolecules within the sampling focus, whether they are involved or not. Specific signatures may dominate, however, and interestingly, the $785-810 \mathrm{~cm}^{-1}$, nucleic acid doublet is prominent in both the studies highlighted here, albeit respectively in the nuclear regions for the action of drugs, and in the cytoplasmic region for nanoparticle toxicity, most likely due to conformational changes in the macromolecules. Differential signatures compared to control (e.g. PCA loading) provide the best visualisation of the characteristic changes due to an exogeneous agent. Regression analyses are appropriate for monitoring systematic evolutions, and although PLSR has largely been used in this work, more sophisticated techniques such as Multiple Component Regression, coupled with Alternating Least Squares approaches [74] may be more appropriate for response cascades. In all representations, the responses are spectrally multivariate, and can have contributions due to conformational changes, increases or decreases in local concentrations of a multitude of different biochemical constituents, including lipids, proteins, carbohydrates and nucleic acids. It is not possible, at present at least, to quantitatively analyse all these changes with molecular specificity. Nevertheless, this study indicates that the spectral signature of combined responses, the "spectralome", is reproducible, consistent and translatable from different cell types and exogenous agents of similar modes of action.

Funding Information. Science Foundation Ireland (11/PI/1108)

\section{References}

1. R. C. Lord, and N. T. Yu, "Laser-excited Raman spectroscopy of biomolecules. I. Native lysozyme and its constituent amino acids," J Mol Biol 50(2), 509-524 (1970).

2. M. C. Tobin, "Raman spectra of crystalline lysozyme, pepsin, and alpha chymotrypsin," Science 161(3836), 68-69 (1968).

3. A. G. Walton, M. J. Deveney, and J. L. Koenig, "Raman spectroscopy of calcified tissue," Calcified Tissue Research 6(1), 162-167 (1970).

4. N.-T. Yu et al., "Single-crystal raman spectra of native insulin: Structures of insulin fibrils, glucagon fibrils, and intact calf lens," Archives of Biochemistry and Biophysics 160(2), 614-622 (1974).

5. G. J. Puppels, and J. Breve, "Whole Cell Studies and Tissue

Characterization by Raman Spectroscopy," in "Biomedical Applications of Spectroscopy", John Wiley and Sons, New York (1996).

6. M. Gniadecka et al., "Distinctive Molecular Abnormalities in Benign and Malignant Skin Lesions: Studies by Raman Spectroscopy," Photochemistry and Photobiology 66(4), 418-423 (1997).

7. C. M. Krishna et al., "Evaluation of the suitability of ex vivo handled ovarian tissues for optical diagnosis by Raman microspectroscopy," Biopolymers 79(5), 269-276 (2005).

8. J. Smith et al., "Raman spectral mapping in the assessment of axillary lymph nodes in breast cancer," Technol Cancer Res Treat 2(4), 327-332 (2003).

9. A. Molckovsky et al., "Diagnostic potential of near-infrared Raman spectroscopy in the colon: differentiating adenomatous from hyperplastic polyps," Gastrointest Endosc 57(3), 396-402 (2003).

10. T. Hollon et al., "Improving the accuracy of brain tumor surgery via Raman-based technology," Neurosurg Focus 40(3), E9 (2016).

11. E. M. Barroso et al., "Water Concentration Analysis by Raman Spectroscopy to Determine the Location of the Tumor Border in Oral Cancer Surgery," Cancer Res 76(20), 5945-5953 (2016).

12. G. Shetty et al., "Raman spectroscopy: elucidation of biochemical changes in carcinogenesis of oesophagus," Br J Cancer 94(10), 1460-1464 (2006).

13. B. Bird et al., "Infrared spectral histopathology (SHP): a novel diagnostic tool for the accurate classification of lung cancer," Lab Invest 92(9), 13581373 (2012)

14. H. J. Byrne et al., "Spectropathology for the next generation: Quo vadis?," Analyst 140(7), 2066-2073 (2015).

15. H. Nawaz et al., "Raman micro spectroscopy study of the interaction of vincristine with A549 cells supported by expression analysis of bcl-2 protein," Analyst 138(20), 6177-6184 (2013).

16. H. Nawaz et al., "Comparison of subcellular responses for the evaluation and prediction of the chemotherapeutic response to cisplatin in lung adenocarcinoma using Raman spectroscopy," Analyst 136(12), 24502463 (2011).

17. P. Knief et al., "Raman spectroscopy - a potential platform for the rapid measurement of carbon nanotube-induced cytotoxicity," Analyst 134(6), 1182-1191 (2009).

18. K. C. Gordon, and C. M. McGoverin, "Raman mapping of pharmaceuticals," Int J Pharm 417(1-2), 151-162 (2011).

19. Q. Tu, and C. Chang, "Diagnostic applications of Raman spectroscopy," Nanomedicine 8(5), 545-558 (2012).

20. Z. Farhane et al., "Cellular discrimination using in vitro Raman micro spectroscopy: the role of the nucleolus," Analyst 140(17), 5908-5919 (2015).

21. Z. Farhane et al., "Raman micro spectroscopy for in vitro drug screening: subcellular localisation and interactions of doxorubicin," Analyst 140(12), 4212-4223 (2015). 
22. E. Efeoglu et al., "Determination of nanoparticle localisation within subcellular organelles in vitro using Raman spectroscopy," Analytical Methods 7(23), 10000-10017 (2015).

23. E. Efeoglu, A. Casey, and H. J. Byrne, "In vitro monitoring of time and dose dependent cytotoxicity of aminated nanoparticles using Raman spectroscopy," Analyst 141(18), 5417-5431 (2016).

24. Y. Oshima et al., "Discrimination analysis of human lung cancer cells associated with histological type and malignancy using Raman spectroscopy," J Biomed Opt 15(1), 017009 (2010).

25. X. Zhang et al., "Characterization of cellular chemical dynamics using combined microfluidic and Raman techniques," Analytical and Bioanalytical Chemistry 390(3), 833-840 (2008).

26. I. Notingher et al., "Spectroscopic study of human lung epithelial cells

(A549) in culture: living cells versus dead cells," Biopolymers 72(4), 230-240 (2003).

27. U. Gala, and H. Chauhan, "Principles and applications of Raman spectroscopy in pharmaceutical drug discovery and development," Expert Opin Drug Discov 10(2), 187-206 (2015).

28. T. Vankeirsbilck et al., "Applications of Raman spectroscopy in pharmaceutical analysis," 21(12), TrAC-Trends in Analytical Chemistry 869877 (2002)

29. D. P. a. P. J. M. Bhumika, "An Overview: Application of Raman Spectroscopy in Pharmaceutical Field," Current Pharmaceutical Analysis 6(2), 131-141 (2010).

30. C. Kallaway et al., "Advances in the clinical application of Raman spectroscopy for cancer diagnostics," Photodiagnosis Photodyn Ther 10(3), 207-219 (2013).

31. I. Pence, and A. Mahadevan-Jansen, "Clinical instrumentation and applications of Raman spectroscopy," Chemical Society Reviews 45(7), 1958-1979 (2016).

32. L. E. Jamieson, and H. J. Byrne, "Vibrational spectroscopy as a tool for studying drug-cell interaction: Could high throughput vibrational spectroscopic screening improve drug development?," Vibrational Spectroscopy 91(16-30 (2017).

33. C. Carvalho et al., "Doxorubicin: the good, the bad and the ugly effect," Curr Med Chem 16(25), 3267-3285 (2009).

34. M. A. Mitry, and J. G. Edwards, "Doxorubicin induced heart failure:

Phenotype and molecular mechanisms," IJC Heart \& Vasculature 10(17-24

(2016).

35. F. S. Carvalho et al., "Doxorubicin-induced cardiotoxicity: from

bioenergetic failure and cell death to cardiomyopathy," Med Res Rev 34(1), 106-135 (2014).

36. J. Hofman et al., "Pharmacokinetic interactions of breast cancer chemotherapeutics with human doxorubicin reductases," Biochem Pharmacol 96(3), 168-178 (2015).

37. J. H. Schiller et al., "Non-Small-Cell Lung Cancer: Then and Now," Journal of Clinical Oncology 31(8), 981-983 (2013).

38. S. Umsumarng et al., "Reversal of human multi-drug resistance leukaemic cells by stemofoline derivatives via inhibition of P-glycoprotein function," Basic Clin Pharmacol Toxicol 116(5), 390-397 (2015).

39. O. Tacar, P. Sriamornsak, and C. R. Dass, "Doxorubicin: an update on anticancer molecular action, toxicity and novel drug delivery systems," J Pharm Pharmacol 65(2), 157-170 (2013).

40. G. Golunski et al., "Pentoxifylline as a modulator of anticancer drug doxorubicin. Part II: Reduction of doxorubicin DNA binding and alleviation of its biological effects," Biochimie 123(95-102 (2016).

41. M. Z. Akhter, and M. R. Rajeswari, "Interaction of doxorubicin with a regulatory element of hmga1 and its in vitro anti-cancer activity associated with decreased HMGA1 expression," J Photochem Photobiol B 141(36-46 (2014).

42. K. Burger et al., "Chemotherapeutic drugs inhibit ribosome biogenesis at various levels," J Biol Chem 285(16), 12416-12425 (2010).

43. R. A. El-Awady et al., "Modulation of DNA damage response and induction of apoptosis mediates synergism between doxorubicin and a new imidazopyridine derivative in breast and lung cancer cells," DNA Repair (Amst) 37(1-11 (2016)

44. S. M. Cutts et al., "The power and potential of doxorubicin-DNA adducts," IUBMB Life 57(2), 73-81 (2005).
45. A. W. El-Kareh, and T. W. Secomb, "Two-mechanism peak concentration model for cellular pharmacodynamics of Doxorubicin," Neoplasia 7(7), 705-713 (2005).

46. A. Zenebergh, R. Baurain, and A. Trouet, "Cellular pharmacology of detorubicin and doxorubicin in L1210 cells," Eur J Cancer Clin Oncol 20(1), 115-121 (1984).

47. Z. Farhane, F. Bonnier, and H. J. Byrne, "An in vitro study of the interaction of the chemotherapeutic drug Actinomycin D with lung cancer cell lines using Raman micro-spectroscopy," J Biophotonics 11(1), (2018). 48. Z. Farhane et al., "In vitro label-free screening of chemotherapeutic drugs using Raman microspectroscopy: Towards a new paradigm of spectralomics," J Biophotonics (2017).

49. Z. Farhane, F. Bonnier, and H.J. Byrne, "Monitoring doxorubicin cellular uptake and trafficking using in vitro Raman microspectroscopy: short and long time exposure effects on lung cancer cell lines," Anal Bioanal Chem 409(5), 1333-1346 (2017).

50. Z. Farhane et al., "Doxorubicin kinetics and effects on lung cancer cell lines using in vitro Raman micro-spectroscopy: binding signatures, drug resistance and DNA repair," J Biophotonics doi:

10.1002/jbio.201700060(n/a-n/a (2017).

51. P. Sandin et al., "High-speed imaging of Rab family small GTPases reveals rare events in nanoparticle trafficking in living cells," ACS Nano 6(2), 1513-1521 (2012).

52. F. Fazlollahi et al., "Polystyrene nanoparticle trafficking across MDCKII," Nanomedicine : nanotechnology, biology, and medicine 7(5), 588-594 (2011).

53. E. Jan et al., "High-content screening as a universal tool for fingerprinting of cytotoxicity of nanoparticles," ACS Nano 2(5), 928-938 (2008).

54. Handbook Of Biological Confocal Microscopy, Springer US (2006). 55. N. M. Radio et al., "Assessment of chemical effects on neurite outgrowth in PC12 cells using high content screening," Toxicol Sci 105(1), 106-118 (2008).

56. B. Ruan et al., "Binding of rapamycin analogs to calcium channels and FKBP52 contributes to their neuroprotective activities," Proc Natl Acad Sci U S A 105(1), 33-38 (2008).

57. G. J. Ding et al., "Characterization and quantitation of NF-kappaB nuclear translocation induced by interleukin-1 and tumor necrosis factoralpha. Development and use of a high capacity fluorescence cytometric system," J Biol Chem 273(44), 28897-28905 (1998).

58. J. M. Zock, "Applications of High Content Screening in Life Science Research," Combinatorial Chemistry \& High Throughput Screening 12(9), 870-876 (2009).

59. K. P. Sarker et al., "ING2 as a novel mediator of transforming growth factor-beta-dependent responses in epithelial cells," J Biol Chem 283(19), 13269-13279 (2008).

60 . U. Liebel, and W. Link, "Meeting report: trends and challenges in high content analysis," Biotechnol J 2(8), 938-940 (2007).

61. D. J. Brayden et al., "High-content analysis for drug delivery and nanoparticle applications," Drug Discovery Today 20(8), 942-957 (2015). 62. A. Casey et al., "Single walled carbon nanotubes induce indirect cytotoxicity by medium depletion in A549 lung cells," Toxicology Letters 179(2), 78-84 (2008).

63. A. L. Holder et al., "Particle-induced artifacts in the MTT and LDH viability assays," Chemical research in toxicology 25(9), 1885-1892 (2012). 64. A. Casey et al., "Spectroscopic analysis confirms the interactions between single walled carbon nanotubes and various dyes commonly used to assess cytotoxicity," Carbon 45(7), 1425-1432 (2007).

65. J. Dorney et al., "Identifying and localizing intracellular nanoparticles using Raman spectroscopy," Analyst 137(5), 1111-1119 (2012).

66. M. E. Keating, F. Bonnier, and H. J. Byrne, "Spectral cross-correlation as a supervised approach for the analysis of complex Raman datasets: the case of nanoparticles in biological cells," Analyst 137(24), 5792-5802 (2012).

67. E. Efeoglu et al., "Label-free, high content screening using Raman microspectroscopy: the toxicological response of different cell lines to amine-modified polystyrene nanoparticles (PS-NH2)," Analyst 142(18), 3500-3513 (2017) 
68. E. Efeoglu, A. Casey, and H. J. Byrne, "Determination of spectral markers of cytotoxicity and genotoxicity using in vitro Raman microspectroscopy: cellular responses to polyamidoamine dendrimer exposure," Analyst 142(20), 3848-3856 (2017).

69. S. P. Mukherjee et al., "Mechanistic studies of in vitro cytotoxicity of poly(amidoamine) dendrimers in mammalian cells," Toxicol Appl

Pharmacol 248(3), 259-268 (2010).

70. M. A. Maher, and H. J. Byrne, "Modification of the in vitro uptake mechanism and antioxidant levels in $\mathrm{HaCaT}$ cells and resultant changes to toxicity and oxidative stress of $\mathrm{G} 4$ and $\mathrm{G} 6$ poly(amidoamine) dendrimer nanoparticles," Analytical and bioanalytical chemistry 408(19), 5295-5307 (2016).

71. C. Wittwehr et al., "How Adverse Outcome Pathways Can Aid the Development and Use of Computational Prediction Models for Regulatory Toxicology", Toxicological Sciences, 155, 326- 336 (2017)

72. M. A. Maher et al., "Numerical simulations of in vitro nanoparticle toxicity - The case of poly(amido amine) dendrimers," Toxicology in Vitro 28(8), 1449-1460 (2014).

73. A. Francis et al., "Label-free pathology by spectrally sliced femtosecond stimulated Raman scattering (SRS) microscopy," PLoS One 12(5), e0178750 (2017).

74. J. Jaumot et al., "A graphical user-friendly interface for MCR-ALS: a new tool for multivariate curve resolution in MATLAB," Chemometrics and Intelligent Laboratory Systems 76(1), 101-110 (2005). 